\title{
Incidence and Implication of Coronary Artery Calcium on Non-gated Chest Computed Tomography Scans: A Large Observational Cohort
}

Charles Haller ${ }^{1}$, Anthony Vandehei ${ }^{1}$, Raymond Fisher ${ }^{1}$, Joshua Boster ${ }^{2}$, Brian Shipley ${ }^{3}$, Christopher Kaatz $^{2}$, Jaclyn Harris ${ }^{2}$, Satoshi R. Shin ${ }^{2}$, Lisa Townsend ${ }^{2}$, Jessica Rouse ${ }^{2}$, Sarah Davis ${ }^{2}$, James Aden ${ }^{4}$, Dustin Thomas ${ }^{1}$

1. Cardiology, Brooke Army Medical Center, Fort Sam Houston, USA 2. Internal Medicine, Brooke Army Medical Center, Fort Sam Houston, USA 3. Internal Medicine, Darnall Army Medical Center, Fort Hood, USA 4. Statistics, Brooke Army Medical Center, Fort Sam Houston, USA

Corresponding author: Dustin Thomas, dustin.m.thomas1@gmail.com

\section{Abstract}

\section{Introduction}

Coronary artery calcification (CAC) scoring is typically performed utilizing non-contrast, electrocardiogram(ECG) gated CT and offers an estimation of cardiovascular (CV) prognosis and risk stratification beyond previously established cardiac risk factors. Coronary calcification can also be assessed during non-gated chest CT, which is significant given the recent recommendations for lung cancer screening by low-dose CT.

\section{Methods}

We retrospectively reviewed 4,953 non-contrast chest CT scans in a single, closed referral tertiary military treatment facility over an 18-month period. Baseline CV outcomes to include myocardial infarction (MI), cerebral vascular accidents (CVA), revascularization with percutaneous coronary intervention (PCI) or coronary artery bypass grafting (CABG), death, or a composite of all major adverse cardiac events (MACE), and baseline CV risk factors were abstracted from an electronic medical record (EMR) review.

\section{Results}

CAC was seen in 3,119 (63\%) patients while 1,834 (27\%) were without CAC. All traditional CV risk factors were more commonly observed in patients with CAC. Unadjusted odds of composite MACE, death, MI, coronary revascularization, and CVA between presence and absence of CAC were as follows: 3.55 [95\% confidence interval (CI): 2.60-4.86, p: <0.0001]; 2.98 (95\% CI: 2.02-4.40, p: <0.0001); 24.42 (95\% CI: 3.36177.6, p: <0.0001); 5.64 (95\% CI: 2.58-12.32, p: <0.0001); and 2.32 (95\% CI: 1.19-4.50, p: 0.0104), respectively. However, after adjusting for baseline risk factors, CAC on non-gated CT was associated only with an increased observed rate of MI (aOR: 38.1, 95\% CI: 4.57-318.2, p: <0.0001) and revascularization (aOR: 5.58, 95\% CI: 2.22-14.0, p; 0.0003).

Received 11/08/2019

Review began 11/12/2019 Review ended 11/19/2019 Published 11/22/2019

\section{() Copyright 2019}

Haller et al. This is an open access article distributed under the terms of the Creative Commons Attribution License CC-BY 3.0., which permits unrestricted use, distribution, and reproduction in any medium, provided the original author and source are credited.

\section{Conclusions}

Findings of CAC on non-gated chest CT may help to recognize patients who are at increased risk of MI and revascularization. Given the expected increase in chest CT utilization among former smokers for lung cancer screening, observed CAC should be reported to ordering providers in order to identify patients at increased risk of these important outcomes.

Categories: Cardiology, Preventive Medicine, Radiology

Keywords: coronary artery calcium, computed tomography

\section{Introduction}

Coronary artery calcium (CAC) is a well-validated risk marker of coronary artery disease (CAD). It adds significant predictive power over traditional risk assessment factors and tools such as carotid media intimal thickness [1-4]. A plethora of data supports a strong relationship between the presence and extent of coronary calcification and clinical outcomes. This is demonstrated among multiple patient cohorts with various risk factors and ethnicities [5].

CAC scoring is traditionally performed utilizing electrocardiogram (ECG) gating with standard reconstruction and acquisition parameters (2-3-mm slice thickness and 120 peak $\mathrm{kV}$ ) as classically described by Agatston et al. [6]. Published data suggests a good correlation among CAC scores between non-gated chest CT scans and formal CAC testing [7-9]. Furthermore, multiple small investigations have reported an increased incidence of adverse cardiovascular (CV) events and death in patients with qualitative coronary calcification on non-contrast, non-gated CT chest scans [10-14]. This is important in light of the recent 
recommendation from the US Preventive Services Task Force (USPSTF), released in 2014, which recommended low-dose CT screening for high-risk current and former smokers [15]. This recommendation carries the possibility of screening an additional 7-10 million patients who are at risk for CV events, with several societies, including the Society of Cardiovascular Computed Tomography (SCCT), recognizing the potential benefit to interpretation and reporting of CAC on these scans [16].

The primary objective of this analysis is to further analyze and inform on the risk for major CV events among patients with and without CAC on non-gated chest CT and assess whether non-gated chest-CTderived $\mathrm{CAC}$ is an independent risk factor for $\mathrm{CV}$ outcomes.

\section{Materials And Methods \\ Study population}

This study involves a single-center, retrospective observational cohort of patients who underwent a noncontrast, non-gated chest CT scan at Brooke Army Medical Center, Fort Sam Houston, TX between January 1, 2011 and June 30, 2012. The local picture archiving and communication system (PACS) was queried for all scans named "CT CHEST W/O CONTRAST" between the dates listed. This query was then refined by the inclusion criteria of a specified patient-age range at the time of scan: between 18 and 80 years.

\section{Coronary artery calcium assessment on non-gated chest CT}

Each scan was reviewed by cardiology staff credentialed in CAC interpretation and cardiology fellows trained in CAC interpretation. Each study was reviewed and marked in a binary fashion as either having or not having coronary calcification, a strictly qualitative assessment. Semi-quantitative analysis of CAC, such as severity, was not reported.

\section{Demographics}

The identified study population was then queried in the local electronic medical record (EMR) for the presence of $\mathrm{CV}$ risk factors and prior use of or the initiation of lipid-lowering medications or aspirin around the time of the imaging study. CV risk factors identified included diabetes mellitus type 2 (DM2), hypertension (HTN) (defined as diagnosis of hypertension by a healthcare provider or $\geqslant 2$ readings of $\geqslant 140 / 90$ $\mathrm{mmHg}$ at outpatient visits), hyperlipidemia (HLP) (defined as diagnosis of hyperlipidemia by a healthcare provider or LDL of $\geqslant 190 \mathrm{mg} / \mathrm{dL}$ ), active or recent smoker (recent smoker defined as any instance of smoking within six months of index scan), or known CAD (defined as any atherosclerotic plaque on coronary computed tomography angiography (CCTA) or invasive coronary angiography (ICA), prior percutaneous coronary intervention (PCI), or prior coronary artery bypass graft $(\mathrm{CABG})$ ).

\section{Outcomes}

Major adverse cardiovascular events (MACE) defined as death, myocardial infarction (MI), cerebrovascular accident (CVA), or revascularization (either PCI or CABG) were abstracted. The instances of death were determined by utilizing the social security death index (SSDI). The remaining MACE outcomes were determined by the EMR chart review. Preliminary data was presented as a conference poster (Conference poster: Fisher R, Haller C, Shipley B, et al.: Incidence and Implication of Coronary Artery Calcium (CAC) on Non-gated Chest CT Scans: A Large Observational Cohort. The Journal of Cardiovascular Computed Tomography. 14th Annual Scientific Meeting of the Society of Cardiovascular Computed Tomography; July 13, 2019).

\section{Statistical analysis}

Continuous variables were analyzed utilizing 2-sided chi-squared testing. The comparison of means was performed using analysis of variance (ANOVA). The comparison of non-normally distributed continuous variables was reported as medians with inter-quartile ranges and analyzed using the Mann-Whitney test. The Kaplan-Meier analysis was performed for event-free survival.

\section{Results}

CAC was diagnosed in 3,119 (63\%) of the patients overall. All traditional CV risk factors (Table 1) were more commonly observed in patients with CAC compared with those without detectable CAC on non-gated chest CT (p: <0.0001). 


\section{Cureus}

\begin{tabular}{|c|c|c|c|}
\hline & CAC $(n=3,119)$ & No CAC $(n=1,834)$ & P-value \\
\hline Age, years & $71( \pm 11)^{\star}$ & $48( \pm 17)^{\star}$ & $<0.0001$ \\
\hline Male gender & $1,793(57.5 \%)$ & $891(48.6 \%)$ & $<0.0001$ \\
\hline Smoker & $1,346(43.2 \%)$ & $493(26.9 \%)$ & $<0.0001$ \\
\hline DM2 & 938 (30.1\%) & 252 (13.7\%) & $<0.0001$ \\
\hline HTN & 2,578 (82.7\%) & $839(45.7 \%)$ & $<0.0001$ \\
\hline HLP & 2,321 (74.4\%) & $704(38.4 \%)$ & $<0.0001$ \\
\hline Known CAD & $1,148(36.8 \%)$ & 178 (9.7\%) & $<0.0001$ \\
\hline Pre-CT statin & $1960(62.8 \%)$ & $440(24.0 \%)$ & $<0.0001$ \\
\hline Pre-CT nonstatin lipid-lowering therapy & 451 (14.5\%) & $106(5.8 \%)$ & $<0.0001$ \\
\hline Pre-CT Aspirin & 1,827 (58.6\%) & $433(23.6 \%)$ & $<0.0001$ \\
\hline
\end{tabular}

TABLE 1: Comparison of demographics and pharmaceutical therapy for patients with coronary artery calcium and those without coronary artery calcium at the time of non-gated CT

CAC: coronary artery calcium; DM2; diabetes mellitus type 2; HTN: hypertension; HLP: hyperlipidemia; CAD: coronary artery disease; CT: computed tomography; n: number

*standard deviation

CAC on non-gated CT was associated with an increased incidence of composite MACE [odds ratio (OR): 3.55 , 95\% CI: 2.60-4.86, p: <0.0001), death (OR: 2.98, 95\% CI: 2.02-4.40, p: <0.0001), MI (OR: 24.42, 95\% CI: 3.36177.6, p: <0.0001), coronary revascularization (OR: 5.64, 95\% CI: 2.58-12.32, p: <0.0001), and CVA (OR: 2.32 , 95\% CI: 1.19-4.50, p: 0.0104 (Table 2). However, after adjusting for baseline risk factors, CAC on non-gated CT was associated only with an increased observed rate of MI [adjuste odds ration (aOR): 38.1, 95\% CI: 4.57318.2, p: <0.0001] and revascularization (aOR: 5.58, 95\% CI: 2.22-14.0, p: 0.0003).

\begin{tabular}{|c|c|c|c|c|c|c|}
\hline Outcome & Unadjusted OR & $95 \% \mathrm{Cl}$ & P-value & Adjusted OR & $95 \% \mathrm{Cl}$ & P-value \\
\hline Composite MACE & 3.55 & $2.60-4.86$ & $<0.0001$ & 1.39 & $0.96-2.02$ & 0.082 \\
\hline Death & 2.98 & $2.02-4.40$ & $<0.0001$ & 0.94 & 0.59-1.49 & 0.779 \\
\hline MI & 24.42 & 3.36-177.6 & $<0.0001$ & 38.1 & $4.57-318.2$ & $<0.0001$ \\
\hline Revascularization & 5.64 & 2.58-12.32 & $<0.0001$ & 5.58 & $2.22-14.0$ & 0.0003 \\
\hline CVA & 2.32 & $1.19-4.50$ & 0.0104 & 0.72 & 0.34-1.52 & 0.3871 \\
\hline
\end{tabular}

TABLE 2: The odds of major adverse cardiovascular event with qualitative coronary artery calcium by non-gated chest computed tomography with adjustment for traditional cardiovascular risk factors

OR: odds ratio; Cl: confidence interval; MACE: major adverse cardiovascular event; MI: myocardial infarction; CVA: cerebrovascular accident

\section{Discussion}

The presence of CAC on non-gated chest CT scans correlated well with traditional cardiac risk factors, including age, male gender, smoking, DM2, HTN, and HLP. These patients with CAC were also more likely to experience MACE, including composite MACE, death, MI, coronary revascularization, and CVA by unadjusted OR. Significantly increased incidence of MI and coronary revascularization remained even after adjusting for baseline risk factors. These findings emphasize the importance of the use of non-gated chest CT scans for the identification of CAC and reinforce the correlation of visual-qualitative CAC with MACE. 
Identification of CAC by non-gated chest CT scans may represent an additional means to risk-stratify patients at risk for MACE. Of patients with visual-qualitative CAC by these studies, only $36.8 \%$ had known CAD. Therefore, $63.2 \%$ of patients with coronary atherosclerosis had not been identified at the time of the scan. These patients perhaps could benefit from cardio-protective pharmacotherapy and lifestyle modification. Approximately $40 \%$ of patients with visual-qualitative CAC by non-contrast chest CT were not on statin, and nearly half of the patients were not on aspirin at the time of the exam.

Although CAC scoring is traditionally performed utilizing ECG gating with standard reconstruction and acquisition parameters, recent publications suggest a good correlation among CAC scores from non-gated chest CT scans and formal CAC testing [7-9]. Several investigations have also demonstrated an increased incidence of MACE associated with CAC, qualitative and quantitative by non-gated chest CT scans [10-14]. Hughes-Austin et al. found a significant increase in the incidence of all-cause mortality for patients with CAC by 6-mm non-gated chest CT scans and demonstrated a correlation with traditional 3-mm ECG-gated CT scans, which remained even after adjusting for traditional CV risk factors [11]. Xie et al. reported a correlation between death and MACE with quantitative and qualitative CAC by non-gated CT in a metaanalysis of five studies [10]. Of note, although trends toward increased death and MACE were noted with CAC, several studies failed to show the significance for these outcomes in subjects with lower quantitative CAC, particularly when adjusted for traditional cardiac risk factors.

This study's findings corroborate the role of qualitative CAC as a predictor of MACE and is strengthened by both a large patient population and subgroup analysis of events with adjustment for traditional cardiac risk factors over a long follow-up period. With the rise in non-gated chest CT scans associated with the USPSTF lung cancer screening recommendations, standardization of reporting CAC for these scans may identify patients at risk for MACE. This provides the opportunity for preventative measures by the ordering provider, such as recommendations for lifestyle modification and prescription of cardio-protective pharmacotherapy to be instituted.

This trial was limited to a strictly visual-qualitative assessment of the presence or absence of CAC. Applying quantitative and semi-quantitative analysis to these non-gated chest CT scans, such as the non-gated Agatston score and other ordinal scoring systems, may further enhance the predictive value for future MACE to levels seen in traditional gated studies. Further limitations include an absence of assessment for variation between investigators and the role such variation may have had on odds, and a failure to compare findings to ECG-gated cardiac CT scans.

\section{Conclusions}

Findings of CAC on non-gated CT may help identify patients who are at increased risk of MI and revascularization. Given the expected increase in chest CT utilization among former smokers for lung cancer screening, observed CAC should be reported to ordering providers in order to identify patients at increased risk of these important outcomes.

\section{Additional Information \\ Disclosures}

Human subjects: Consent was obtained by all participants in this study. Brooke Army Medical Center issued approval C.2017.216d. Retrospective study approved for research and publication by the Brooke Army Medical Center Institutional Review Board and the Public Affairs Office. Animal subjects: All authors have confirmed that this study did not involve animal subjects or tissue. Conflicts of interest: In compliance with the ICMJE uniform disclosure form, all authors declare the following: Payment/services info: All authors have declared that no financial support was received from any organization for the submitted work. Financial relationships: All authors have declared that they have no financial relationships at present or within the previous three years with any organizations that might have an interest in the submitted work. Other relationships: All authors have declared that there are no other relationships or activities that could appear to have influenced the submitted work.

\section{Acknowledgements}

The views expressed herein are those of the authors and do not reflect the official policy or position of Brooke Army Medical Center, the U.S. Army Medical Department, the U.S. Army Office of the Surgeon General, the Department of the Army, Department of the Air Force, or U.S. Government.

\section{References}

1. Budoff MJ, Mohlenkamp S, McClelland R, et al.: A comparison of outcomes with coronary artery calcium scanning in unselected populations: the Multi-Ethnic Study of Atherosclerosis (MESA) and Heinz Nixdorf RECALL study (HNR). J Cardiovasc Comput Tomogr. 2013, 7:182-191. 10.1016/j.jcct.2013.05.009

2. Folsom AR, Kronmal RA, Detrano RC, et al.: Coronary artery calcification compared with carotid intimamedia thickness in the prediction of cardiovascular disease incidence: the Multi-Ethnic Study of Atherosclerosis (MESA). Arch Intern Med. 2008, 168:1333-1339. 10.1001/archinte.168.12.1333 
3. Erbel R, Mohlenkamp S, Moebus S, et al.: Coronary risk stratification, discrimination, and reclassification improvement based on quantification of subclinical coronary atherosclerosis: the Heinz Nixdorf Recall study. J Am Coll Cardiol. 2010, 56:1397-1406. 10.1016/j.jacc.2010.06.030

4. Yeboah J, McClelland RL, Polonsky TS, et al.: Comparison of novel risk markers for improvement in cardiovascular risk assessment in intermediate-risk individuals. JAMA. 2012, 308:788-795. 10.1001/jama.2012.9624

5. Blaha MJ, Budoff MJ, DeFilippis AP, et al.: Associations between C-reactive protein, coronary artery calcium, and cardiovascular events: implications for the JUPITER population from MESA, a population-based cohort study. Lancet. 2011, 378:684-692. 10.1016/S0140-6736(11)60784-8

6. Agatston AS, Janowitz WR, Hildner FJ, Zusmer NR, Viamonte M Jr, Detrano R: Quantification of coronary artery calcium using ultrafast computed tomography. J Am Coll Cardiol. 1990, 15:827-832. 10.1016/07351097(90)90282-t

7. Wu MT, Yang P, Huang YL, Chen JS, Chuo CC, Yeh C, Chang RS: Coronary arterial calcification on low-dose ungated MDCT for lung cancer screening: concordance study with dedicated cardiac CT. AJR Am J Roentgenol. 2008, 190:923-928. 10.2214/AJR.07.2974

8. Takx RA, de Jong PA, Leiner T, et al.: Automated coronary artery calcification scoring in non-gated chest CT: agreement and reliability. PLoS One. 2014, 9:e91239. Accessed: November 21, 2019: 10.1371/journal.pone.0091239

9. Htwe Y, Cham MD, Henschke CI, et al.: Coronary artery calcification on low-dose computed tomography: comparison of Agatston and ordinal scores. Clin Imaging. 2015, 39:799-802. 10.1016/j.clinimag.2015.04.006

10. Xie X, Zhao Y, de Bock GH, de Jong PA, Mali WP, Oudkerk M, Vliegenthart R: Validation and prognosis of coronary artery calcium scoring in nontriggered thoracic computed tomography: systematic review and meta-analysis. Circ Cardiovasc Imaging. 2013, 6:514-521. 10.1161/CIRCIMAGING.113.000092

11. Hughes-Austin JM, Dominguez A 3rd, Allison MA, et al.: Relationship of coronary calcium on standard chest ct scans with mortality. JACC Cardiovasc Imaging. 2016, 9:152-159. 10.1016/j.jcmg.2015.06.030

12. Mets OM, Vliegenthart R, Gondrie MJ, et al.: Lung cancer screening CT-based prediction of cardiovascular events. JACC Cardiovasc Imaging. 2013, 6:899-907. 10.1016/j.jcmg.2013.02.008

13. Chiles C, Duan F, Gladish GW, et al.: Association of coronary artery calcification and mortality in the National Lung Screening Trial: a comparison of three scoring methods. Radiology. 2015, 276:82-90. 10.1148/radiol.15142062

14. Shemesh J, Henschke CI, Shaham D, et al.: Ordinal scoring of coronary artery calcifications on low-dose CT scans of the chest is predictive of death from cardiovascular disease. Radiology. 2010, 257:541-548. 10.1148/radiol.10100383

15. Moyer VA; U.S. Preventive Services Task Force: Screening for lung cancer: U.S. Preventive Services Task Force recommendation statement. Ann Intern Med. 2014, 160:330-338. 10.7326/M13-2771

16. Hecht HS, Cronin P, Blaha MJ, et al.: 2016 SCCT/STR guidelines for coronary artery calcium scoring of noncontrast noncardiac chest CT scans: a report of the Society of Cardiovascular Computed Tomography and Society of Thoracic Radiology. J Cardiovasc Comput Tomogr. 2017, 11:74-84. 10.1016/j.jcct.2016.11.003 